\title{
选择性合成双[噻吩并[2,3-0]嘧啶-4(3H)-酮]
}

\author{
方正东* 吕鉴泉卫杨超＼cjkstart袁叔雅
}

(湖北师范学院化学与环境工程学院 黄石 435002)

\begin{abstract}
摘要 以 2-氨基-4-三氟甲基-5-甲基-噻吩-3-羧酸乙酯(1)为起始原料制得膦亚胺 2 . 在碳酸钾的催化下, 膦亚胺 2 与芳 基异氭酸酯和伯二胺的氮杂 Wittig 反应制得嘧啶环上 2,2'取代的双[噻吩并[2,3- $d$ ] 嘧啶-4(3H)-酮] 3; 膦亚胺 2 与烷基异 氰酸酯和伯二胺的氮杂 Wittig 反应制得嘧啶环上 3,3'取代的双[噻吩并[2,3- $d$ 嘧啶-4(3H)-酮] 4. 化合物 3 的核磁共振氢 谱表明关环反应在嘧啶环的 2,2'位; 化合物 4 的核磁共振氢谱表明关环反应在嘧啶环的 3,3'位. 对合成反应机理的推导 及目标产物核磁共振氢谱数据的分析解释了此合成反应的选择性.
\end{abstract}

关键词＼cjkstart噻吩并 $[2,3-d]$ 嘧啶-4(3H)-酮; 异氭酸酯; 膦亚胺; 伯二胺; 选择性合成

\section{Selective Synthesis of Bis(thieno[2,3-d]pyrimidin-4(3H)-ones)}

\author{
Fang, Zhengdong* Lü, Jianquan Wei, Yangchao Yuan, Shuya \\ (College of Chemistry and Environmental Engineering, Hubei Normal University, Huangshi 43502)
}

\begin{abstract}
The iminophosphorane 2 was obtained using ethyl 2-amino-4-trifluoromethyl-5-methyl-thiophene-3-carboxylate (1) as starting materials. Further aza-Wittig reactions of iminophosphorane 2 with aromatic isocyanate and primary diamine produced 2,2'-disubstituted bis(thieno[2,3- $d$ ]pyrimidin-4(3H)-ones) 3 in the presence of catalytic amounts of potassium carbonate in $70 \% \sim 87 \%$ yields. Aza-Wittig reactions of iminophosphorane 2 with alkyl isocyanate and primary diamine produced 3,3'-disubstituted bis(thieno[2,3-d]pyrimidin-4(3H)-ones) 4 in 38\% 57\% yields. ${ }^{1} \mathrm{H}$ NMR of compound 3 revealed the exclusive selectivity of these ring closures toward the 2,2'-disubstituted position of pyrimidine moiety, and ${ }^{1} \mathrm{H}$ NMR of compound 4 revealed the exclusive selectivity of these ring closures toward the 3,3'-disubstituted position of pyrimidine moiety. The reaction mechanism was also proposed for the regioselectivity.
\end{abstract}

Keywords thieno[2,3- $d]$ pyrimidin-4(3H)-one; isocyanate; iminophosphorane; primary diamine; selective synthesis

选择性合成反应是有机合成领域以化学手段制备所 需化学构型物质的有效方法, 可以减少副产物的生成, 在分子设计、药物合成等方面具有重要的作用. 近年来, Wittig 反应由于在合成含氮杂环化合物中的简捷和高效 性而引起化学研究者的极大兴趣 ${ }^{[1 \sim 3]}$, 合成了一系列具有 良好生物活性和药理活性的含氮杂环化合物 ${ }^{[4-6]}$. 当膦叶 立德与异氰酸酯反应时可以生成碳二亚胺, 碳二亚胺分 子结构中的氮杂累积二烯结构 $(\mathrm{N}=\mathrm{C}=\mathrm{N})$, 使它具有很 好的化学活性, 可以和胺、醇、酚等含活泼氢化合物发 生亲核反应，反应中间体经催化关环便生成具有嘧啶结 构的化合物 ${ }^{[7 \sim 9]}$. 与仲胺、醇、酚等不一样 ${ }^{[10,11]}$, 伯胺与 碳二亚胺反应后, 存在两个氮原子竞争反应, 能选择性 关环得到不同的反应产物 ${ }^{[12]}$. 对于这种多组份或多官能 团参与的反应, 如何进行反应的选择性控制是提高合成
效率的重要研究课题. 本课题组运用氮杂 Wittig 反应合 成了一系列桥联的噻吩并 $[2,3-d]$ 嘧啶-4(3H)-酮, 这里进 一步报道应用膦亚胺与异氧酸酯和伯二胺反应选择性 合成嘧啶环上 $2,2^{\prime}$ 位或 3,3'位取代的双[塞吩并 [2,3- $d$ 嘧 啶-4(3H)-酮]. 实验说明, 在氮杂 Wittig 反应中, 如果膦 亚胺结构确定，当采用不同取代基的异氧酸酯或伯二胺 进行反应时，可以实现其关环反应的选择性调控，得到 预期化学结构的产物. 该方法原料易得，操作简便，反应 选择性好. 合成路线见 Scheme 1.

\section{1 实验部分}

\section{1 仪器及试剂}

熔点测定采用北京泰克 X-4 型熔点仪, 温度计未经

\footnotetext{
* E-mail: zdfangluo@sina.com

Received May 19, 2011; revised July 11, 2011; accepted September 1, 2011.

Project supported by the Natural Science Foundation of Hubei Province (No. 2010CDA061).

湖北省自然科学基金(No. 2010CDA061)资助项目.
} 


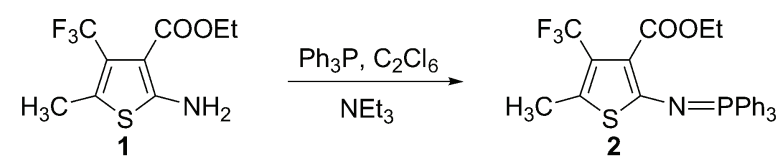<smiles>CCOC(=O)c1c(/N=[P+]\c2ccccc2)sc(C)c1C(F)(F)F</smiles><smiles>[Z]Nc1nc2sc(C)c(C)c2c(=O)n1[Al]Nc1nc2sc(C)c(C(F)(F)F)c2c(=O)n1[Al]</smiles>

3<smiles>[R]Nc1nc2sc(C)c(C(F)(F)F)c2c(=O)n1[Z]Cn1c(N[R])nc2sc(C)c(C(F)(F)F)c2c1=O</smiles>

Scheme 1

校正; 红外以 Nicolet $7500 \mathrm{NXR}$ 红外光谱仪测定 $(\mathrm{KBr}$ 压 片); ${ }^{1} \mathrm{H}$ NMR 用 Varian Mercury 400 型核磁共振仪测定, TMS 为内标; MS 使用 Finnigan LCQ Advantage MAX 质 谱仪测定; 元素分析使用 Perkin-Elmer CHN 2400 元素分 析仪测定.

所用试剂均为分析纯.

\section{2 目标产物双[噻吩并[2,3-d]嘧啶-4(3H)-酮]的制备}

中间体膦亚胺 2 的制备参照文献[13]方法进行, 产 率 $85 \%$, m.p. $153 \sim 154{ }^{\circ} \mathrm{C}$. 然后, 在 $0 \sim 5{ }^{\circ} \mathrm{C}$ 及干燥氮气 保护下, 将不同取代基的异氰酸酯(7 mmol)滴加到膦亚 胺 2 (3.6 g, $7 \mathrm{mmol}$ )的无水四氢呋喃(THF)溶液中, 控制 反应温度 $0 \sim 35{ }^{\circ} \mathrm{C}$, 摚拌反应, TLC 跟踪至反应物膦亚 胺 2 消失. 滴加相应伯二胺 (3 mmol)的无水 THF 溶液, 加入催化量的无水碳酸钾, 继续加热反应 $4 \sim 10 \mathrm{~h}$. 减压 蒸去溶剂, 残余物以二氯甲烷-乙醇混合溶剂(体积比 $1: 1)$ 重结晶, 得到相应的双 [噻吩并 [2,3- $d$ ] 嘧啶-4(3H)酮] 3 或 4. 合成化合物见表 1 .

3a: 白色晶体. m.p. $218 \sim 219{ }^{\circ} \mathrm{C} ;{ }^{1} \mathrm{H}$ NMR $\left(\mathrm{CDCl}_{3}\right.$, $400 \mathrm{MHz}) \delta: 2.35\left(\mathrm{~s}, 6 \mathrm{H}, 2 \mathrm{CH}_{3}\right), 3.14(\mathrm{q}, J=7.0 \mathrm{~Hz}, 4 \mathrm{H}$, $\left.2 \mathrm{NCH}_{2}\right), 4.31(\mathrm{t}, J=6.0 \mathrm{~Hz}, 2 \mathrm{H}, 2 \mathrm{NH}), 7.12 \sim 7.45(\mathrm{~m}$, 10H, Ar-H); IR (KBr) v: $3320(\mathrm{~N}-\mathrm{H}), 1678(\mathrm{C}=\mathrm{O})$, 1557, 1456, $1349 \mathrm{~cm}^{-1}$; ESI-MS m/z: $677.1\left(\mathrm{M}^{+}+\mathrm{H}\right)$. Anal. calcd for $\mathrm{C}_{30} \mathrm{H}_{22} \mathrm{~N}_{6} \mathrm{O}_{2} \mathrm{~S}_{2} \mathrm{~F}_{6}$ : C 53.25, H 3.28, N 12.42; found $\mathrm{C} 53.17, \mathrm{H} 3.25, \mathrm{~N} 12.43$.

3b: 白色晶体. m.p. 253 254 ${ }^{\circ} \mathrm{C}$; ${ }^{1} \mathrm{H}$ NMR $\left(\mathrm{CDCl}_{3}\right.$, $400 \mathrm{MHz}) \delta: 2.35\left(\mathrm{~s}, 6 \mathrm{H}, 2 \mathrm{CH}_{3}\right), 6.32(\mathrm{~s}, 2 \mathrm{H}, 2 \mathrm{NH}), 7.40 \sim$ 7.52 (m, 10H, Ar-H), 7.59 (s, 4H, Ph-H); IR (KBr) v: 3322 $(\mathrm{N}-\mathrm{H}), 1676(\mathrm{C}=\mathrm{O}), 1556,1516,1438 \mathrm{~cm}^{-1}$; ESI-MS
表 1 目标化合物 $\mathbf{3}$ 和 $\mathbf{4}$ 的合成

Table 1 Preparation of the title compounds 3 and 4

\begin{tabular}{|c|c|c|c|}
\hline Compd. & $\operatorname{Ar}(\mathrm{R})$ & Z & Yield/\% \\
\hline $3 \mathbf{a}$ & $\mathrm{Ph}$ & $-\mathrm{CH}_{2} \mathrm{CH}_{2}-$ & 84 \\
\hline $3 b$ & $\mathrm{Ph}$ & & 87 \\
\hline $3 c$ & 4- $\mathrm{MeC}_{6} \mathrm{H}_{4}$ & $-\mathrm{CH}_{2} \mathrm{CH}_{2}-$ & 80 \\
\hline 3d & 4- $\mathrm{MeC}_{6} \mathrm{H}_{4}$ & & 70 \\
\hline $3 e$ & $4-\mathrm{ClC}_{6} \mathrm{H}_{4}$ & $-\mathrm{CH}_{2} \mathrm{CH}_{2}-$ & 72 \\
\hline $3 f$ & $4-\mathrm{ClC}_{6} \mathrm{H}_{4}$ & & 71 \\
\hline $4 a$ & $i$-Propyl & $-\mathrm{CH}_{2} \mathrm{CH}_{2}-$ & 57 \\
\hline $4 b$ & $i$-Propyl & & 42 \\
\hline $4 c$ & n-Butyl & $-\mathrm{CH}_{2} \mathrm{CH}_{2}-$ & 51 \\
\hline $4 d$ & $n$-Butyl & & 38 \\
\hline
\end{tabular}

$m / z: 725.4\left(\mathrm{M}^{+}+\mathrm{H}\right)$. Anal. calcd for $\mathrm{C}_{34} \mathrm{H}_{22} \mathrm{~N}_{6} \mathrm{O}_{2} \mathrm{~S}_{2} \mathrm{~F}_{6}$ : C 56.35, H 3.06, N 11.60; found C 56.37, H 3.05, N 11.49.

3c: 白色晶体. m.p. $238 \sim 239{ }^{\circ} \mathrm{C}$; ${ }^{1} \mathrm{H}$ NMR $\left(\mathrm{CDCl}_{3}\right.$, $400 \mathrm{MHz}) \delta: 2.35\left(\mathrm{~s}, 6 \mathrm{H}, 2 \mathrm{CH}_{3}\right), 2.41\left(\mathrm{~s}, 6 \mathrm{H}, 2 \mathrm{Ph}-\mathrm{CH}_{3}\right)$, 3.14 (q, $J=7.0 \mathrm{~Hz}, 4 \mathrm{H}, 2 \mathrm{NCH}_{2}$ ), 4.30 (t, $J=7.0 \mathrm{~Hz}, 2 \mathrm{H}$, 2NH), $7.12 \sim 7.45(\mathrm{~m}, 8 \mathrm{H}, \mathrm{Ar}-\mathrm{H})$; IR (KBr) v: $2965(\mathrm{~N}-\mathrm{H})$, $1687(\mathrm{C}=\mathrm{O}), 1541,1456,1347 \mathrm{~cm}^{-1}$; ESI-MS m/z: 705.1 $\left(\mathrm{M}^{+}+\mathrm{H}\right)$. Anal. calcd for $\mathrm{C}_{32} \mathrm{H}_{26} \mathrm{~N}_{6} \mathrm{O}_{2} \mathrm{~S}_{2} \mathrm{~F}_{6}$ : C 54.54, H 3.72, $\mathrm{N} 11.93$; found C 54.51, H 3.75, N 12.01.

3d: 白色晶体. m.p. $262 \sim 263{ }^{\circ} \mathrm{C} ;{ }^{1} \mathrm{H}$ NMR $\left(\mathrm{CDCl}_{3}\right.$, $400 \mathrm{MHz}) \delta: 2.35\left(\mathrm{~s}, 6 \mathrm{H}, 2 \mathrm{CH}_{3}\right), 2.41\left(\mathrm{~s}, 6 \mathrm{H}, 2 \mathrm{PhCH}_{3}\right)$, $6.32(\mathrm{~s}, 2 \mathrm{H}, 2 \mathrm{NH}), 7.10 \sim 7.32(\mathrm{~m}, 8 \mathrm{H}, \mathrm{Ar}-\mathrm{H}), 7.57$ (s, 4H, $\mathrm{Ph}-\mathrm{H})$; IR (KBr) v: $3361(\mathrm{~N}-\mathrm{H}), 1687(\mathrm{C}=\mathrm{O})$, 1540, 1516, $1342 \mathrm{~cm}^{-1}$; ESI-MS m/z: $753.4\left(\mathrm{M}^{+}+\mathrm{H}\right)$. Anal. calcd for $\mathrm{C}_{36} \mathrm{H}_{26} \mathrm{~N}_{6} \mathrm{O}_{2} \mathrm{~S}_{2} \mathrm{~F}_{6}$ : C 57.44, H 3.48, N 11.16; found C 57.37, H 3.45, N 11.19.

3e: 白色晶体. m.p. $>300{ }^{\circ} \mathrm{C} ;{ }^{1} \mathrm{H}$ NMR $\left(\mathrm{CDCl}_{3}, 400\right.$ $\mathrm{MHz}) \delta: 2.32\left(\mathrm{~s}, 6 \mathrm{H}, 2 \mathrm{CH}_{3}\right), 3.07(\mathrm{q}, J=7.0 \mathrm{~Hz}, 4 \mathrm{H}$, $\left.2 \mathrm{NCH}_{2}\right), 4.34(\mathrm{t}, J=7.0 \mathrm{~Hz}, 2 \mathrm{H}, 2 \mathrm{NH}), 7.24 \sim 7.47(\mathrm{~m}, 8 \mathrm{H}$, Ar-H); IR (KBr) v: $3338(\mathrm{~N}-\mathrm{H}), 1675(\mathrm{C}=\mathrm{O})$, 1532, 1446, $1339 \mathrm{~cm}^{-1}$; ESI-MS m/z: $745.1\left(\mathrm{M}^{+}+\mathrm{H}\right)$. Anal. calcd for $\mathrm{C}_{30} \mathrm{H}_{20} \mathrm{~N}_{6} \mathrm{O}_{2} \mathrm{~S}_{2} \mathrm{~F}_{6} \mathrm{Cl}_{2}$ : C 48.33, H 2.70, N 11.27; found $\mathrm{C} 48.39, \mathrm{H} 2.75, \mathrm{~N} 11.22$.

3f: 白色晶体, m.p. $>300{ }^{\circ} \mathrm{C} ;{ }^{1} \mathrm{H}$ NMR $\left(\mathrm{CDCl}_{3}, 400\right.$ $\mathrm{MHz}) \delta: 2.31\left(\mathrm{~s}, 6 \mathrm{H}, 2 \mathrm{CH}_{3}\right), 6.75(\mathrm{~s}, 2 \mathrm{H}, 2 \mathrm{NH}), 7.41 \sim 7.52$ (m, 8H, Ar-H), 7.30 (s, 4H, Ph-H); IR (KBr) v: $3328(\mathrm{~N}-$ 
H), $1668(\mathrm{C}=\mathrm{O}), 1543,1536,1443 \mathrm{~cm}^{-1}$; ESI-MS $\mathrm{m} / \mathrm{z}$ : $793.2\left(\mathrm{M}^{+}+\mathrm{H}\right)$. Anal. calcd for $\mathrm{C}_{34} \mathrm{H}_{20} \mathrm{~N}_{6} \mathrm{O}_{2} \mathrm{~S}_{2} \mathrm{~F}_{6} \mathrm{Cl}_{2}$ : C 51.46, H 2.54, N 10.59. found C 51.38, H 2.55, N 10.52 .

4a: 白色晶体. m.p. $>300{ }^{\circ} \mathrm{C} ;{ }^{1} \mathrm{H}$ NMR $\left(\mathrm{CDCl}_{3}, 400\right.$ MHz) $\delta: 1.04\left(\mathrm{~d}, J=6.8 \mathrm{~Hz}, 12 \mathrm{H}, 4 \mathrm{CH}_{3}\right), 2.35(\mathrm{~s}, 6 \mathrm{H}$, $\left.2 \mathrm{CH}_{3}\right), 3.14\left(\mathrm{t}, J=7.0 \mathrm{~Hz}, 4 \mathrm{H}, 2 \mathrm{CH}_{2}\right), 4.64 \sim 4.70(\mathrm{~m}, 2 \mathrm{H}$, $2 \mathrm{NCH}), 5.31(\mathrm{~d}, J=7.0 \mathrm{~Hz}, 2 \mathrm{H}, 2 \mathrm{NH})$; IR (KBr) $v: 3330$ $(\mathrm{N}-\mathrm{H}), 1673(\mathrm{C}=\mathrm{O}), 1547,1514,1347 \mathrm{~cm}^{-1}$; ESI-MS m/z: $609.6\left(\mathrm{M}^{+}+\mathrm{H}\right)$. Anal. calcd for $\mathrm{C}_{24} \mathrm{H}_{26} \mathrm{~N}_{6} \mathrm{O}_{2} \mathrm{~S}_{2} \mathrm{~F}_{6}$ : C 47.36, H 4.31, N 13.81; found C 47.24, H 4.25, N 13.83.

4b: 白色晶体. m.p. $>300{ }^{\circ} \mathrm{C} ;{ }^{1} \mathrm{H} \mathrm{NMR}\left(\mathrm{CDCl}_{3}, 400\right.$ MHz) $\delta: 1.05\left(\mathrm{~d}, J=6.8 \mathrm{~Hz}, 12 \mathrm{H}, 4 \mathrm{CH}_{3}\right), 2.35(\mathrm{~s}, 6 \mathrm{H}$, $\left.2 \mathrm{CH}_{3}\right), 4.04 \sim 4.10(\mathrm{~m}, 2 \mathrm{H}, 2 \mathrm{NCH}), 5.32(\mathrm{~d}, J=7.2 \mathrm{~Hz}$, 2H, 2NH), 7.56 (s, 4H, Ph-H); IR (KBr) v: $3324(\mathrm{~N}-\mathrm{H})$, $1675(\mathrm{C}=\mathrm{O}), 1574,1498,1335 \mathrm{~cm}^{-1}$; ESI-MS m/z: 657.4 $\left(\mathrm{M}^{+}+\mathrm{H}\right)$. Anal. calcd for $\mathrm{C}_{28} \mathrm{H}_{26} \mathrm{~N}_{6} \mathrm{O}_{2} \mathrm{~S}_{2} \mathrm{~F}_{6}: \mathrm{C}$ 51.21, H 3.99, N 12.80; found C 51.17, H 4.03, N 12.69 .

4c: 白色晶体. m.p. $>300{ }^{\circ} \mathrm{C} ;{ }^{1} \mathrm{H}$ NMR $\left(\mathrm{CDCl}_{3}, 400\right.$ MHz) $\delta: 1.06\left(\mathrm{t}, J=7.2 \mathrm{~Hz}, 6 \mathrm{H}, 2 \mathrm{CH}_{3}\right), 1.13 \sim 1.21(\mathrm{~m}, 4 \mathrm{H}$, $\left.2 \mathrm{CH}_{2}\right), 1.43 \sim 1.50\left(\mathrm{~m}, 4 \mathrm{H}, 2 \mathrm{CH}_{2}\right), 2.35$ (s, $\left.6 \mathrm{H}, 2 \mathrm{CH}_{3}\right), 3.14$ (q, $J=7.0 \mathrm{~Hz}, 4 \mathrm{H}, 2 \mathrm{NCH}_{2}$ ), 5.37 (t, $J=7.0 \mathrm{~Hz}, 2 \mathrm{H}, 2 \mathrm{NH}$ ), $4.04 \sim 4.10\left(\mathrm{t}, J=6.2 \mathrm{~Hz}, 2 \mathrm{H}, 2 \mathrm{CH}_{2}\right) ; \mathrm{IR}(\mathrm{KBr}) v: 3325(\mathrm{~N}-$ H), $1687(\mathrm{C}=\mathrm{O}), 1547,1516,1349 \mathrm{~cm}^{-1}$; ESI-MS $m / z$ : $637.7\left(\mathrm{M}^{+}+\mathrm{H}\right)$. Anal. calcd for $\mathrm{C}_{26} \mathrm{H}_{30} \mathrm{~N}_{6} \mathrm{O}_{2} \mathrm{~S}_{2} \mathrm{~F}_{6}$ : C 49.05, H 4.75, N 13.20; found C 49.12, H 4.71, N 13. 26.

4d: 白色晶体. m.p. $>300{ }^{\circ} \mathrm{C} ;{ }^{1} \mathrm{H} \mathrm{NMR}\left(\mathrm{CDCl}_{3}, 400\right.$ MHz) $\delta: 1.06\left(\mathrm{t}, J=7.2 \mathrm{~Hz}, 6 \mathrm{H}, 2 \mathrm{CH}_{3}\right), 1.13 \sim 1.21(\mathrm{~m}$, $\left.4 \mathrm{H}, 2 \mathrm{CH}_{2}\right), 1.43 \sim 1.50\left(\mathrm{~m}, 4 \mathrm{H}, 2 \mathrm{CH}_{2}\right), 2.35\left(\mathrm{~s}, 6 \mathrm{H}, 2 \mathrm{CH}_{3}\right.$ ), 3.10 (q, $J=6.8 \mathrm{~Hz}, 4 \mathrm{H}, 2 \mathrm{NCH}_{2}$ ), 5.32 (t, $J=7.0 \mathrm{~Hz}, 2 \mathrm{H}$, 2NH), 7.51 (s, 4H, Ph-H); IR (KBr) v: $2976 \quad(\mathrm{~N}-\mathrm{H})$, $1670(\mathrm{C}=\mathrm{O}), 1545,1506,1340 \mathrm{~cm}^{-1}$; ESI-MS $m / z$ : 685.7 $\left(\mathrm{M}^{+}+\mathrm{H}\right)$. Anal. calcd for $\mathrm{C}_{30} \mathrm{H}_{30} \mathrm{~N}_{6} \mathrm{O}_{2} \mathrm{~S}_{2} \mathrm{~F}_{6}: \mathrm{C} 52.62, \mathrm{H}$ 4.42, N 12.27; found C 52.37, H 4.35, N 12.43.

\section{2 结果与讨论}

\section{1 合成反应}

在目标化合物的合成中, 含 $\mathrm{P}=\mathrm{X}$ 双键的五价有机 膦化合物(膦亚胺)与异氭酸酯反应制得碳二亚胺, 反应 过程经过四元环过渡态 ${ }^{[14 ~ 16]}$, 反应机理参见文献[13].

在此反应中, 烷基异氧酸酯与膦亚胺的反应较芳基 异氰酸酯慢. 在 $0 \sim 5{ }^{\circ} \mathrm{C}$ 下, 芳基异氧酸酯与膦亚胺的 反应需 4 6 h, 而烷基异氧酸酯与膦亚胺的反应需 8〜 $16 \mathrm{~h}$. 为此, 实验时烷基异氧酸酯与膦亚胺的反应温度 应适当提高. 当温度控制在 $30 \sim 35{ }^{\circ} \mathrm{C}$ 时, 反应可在 $4 \sim$ $6 \mathrm{~h}$ 完成.
伯二胺与碳二亚胺亲核加成得到胍类中间体. 此加 成反应受伯二胺空间体积大小的影响, 空间位越阻大, 活性越低, 反应速率就越小.

在碳酸钾催化下，胍类中间体进行分子内反应而成 环，关环反应是由于氨基氮原子对 $\beta$ 位酯基的亲核进攻. 此过程中, 由于胍分子内存在两个活泼亚氨基 $(\mathrm{NH})$, 一 个是原碳二亚胺 $(\mathrm{N}=\mathrm{C}=\mathrm{N})$ 基团还原生成的，与芳环或 烷基连接; 一个是原伯二胺上的，与伯二胺取代基(Z)连 接. 这两个活泼亚氨基的区域位置可以通过 $\mathrm{C}-\mathrm{N}$ 单键 的旋转而变化, 都能与 $\beta$ 位酯基进行亲核反应, 见 Scheme 2 .

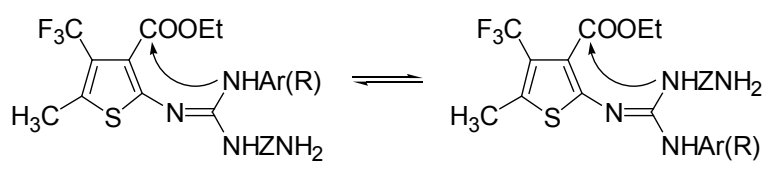

Scheme 2

因此，关环反应是这两个活泼亚氨基的竞争反应， 分子中取代基不同，就存在不同的选择性. 实验结果说 明，亲核反应受电子效应和体积效应控制. 当采用芳基 异氧酸酯进行反应时, 生成的碳二亚胺上的取代基为芳 环，在碳酸钾作用下，与芳环连接的亚氨基 $(\mathrm{NH})$ 脱去质 子 $\left(\mathrm{H}^{+}\right)$, 中间体 $\mathbf{A}$ 由于共轭稳定, 更容易生成, 从而有 利于此亚氨基氮原子对 $\beta$ 位酯基亲核进攻,

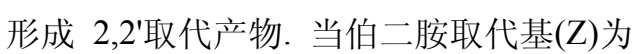
苯环时, 它的空间位阻大于芳环, 也是形 $\mathrm{N}==_{\mathrm{NHZNH}}^{-\mathrm{N}-\mathrm{Ar}}$ A 成 2,2 '取代产物.

当采用烷基异氧酸酯进行反应时, 生成的碳二亚胺 上的取代基为烷基, 而伯二胺取代基 $(\mathrm{Z})$ 为苯环时, 与此 苯环连接的亚氨基 $(\mathrm{NH})$ 脱去质子 $\left(\mathrm{H}^{+}\right)$, 中间体 $\mathbf{B}$ 由于共 轭稳定, 更容易生成, 从而有利于此亚氨基氮原子对 $\beta$ 位酯基亲核进攻, 形成 3,3'取代产物. 当碳二亚胺 $(\mathrm{N}=$ $\mathrm{C}=\mathrm{N}$ )上的烷基为异丙基或正丁基，伯二胺取代基( $\mathrm{Z}$ )为 $\mathrm{CH}_{2} \mathrm{CH}_{2}$ 时, 氨基上连接的都有是烷基, 反应主要受体 积效应控制. 伯二胺取代基 $(\mathrm{Z})$ 体积小, 有利于此氨基氮原子对 $\beta$ 位酯基亲核进 攻, 也形成 3,3'取代产物. 可能的反应机 $\mathrm{N}=\mathrm{NHR}_{\mathrm{NH}}^{\mathrm{N}-\mathrm{ZNH}_{2}}$ 理见 Scheme 3.

\section{2 双[噻吩并[2,3- $d]$ 嘧啶-4(3H)-酮]的波谱特征}

关环反应的选择性可以在产物的 ${ }^{1} \mathrm{H}$ NMR 中得到证 实. 如化合物 3a, 在 $\delta 3.72$ 处有一四重峰, 为 $\mathrm{NCH}_{2}$ 质子 信号; 在 $\delta 4.31$ 处有一三重峰, 为氨基 $(\mathrm{NH})$ 质子信号, 相 比 $4 \mathbf{a}$ 的亚氨基 $(\mathrm{NH})$ 质子信号更趋向高场, 证实桥联取代 在 3 位. 而化合物 $\mathbf{4 a}$, 在 $\delta 5.31$ 处有一双重峰, 为亚氨基 $(\mathrm{NH})$ 质子信号; 在 $\delta 4.64 \sim 4.70$ 处有一多重峰, 为基团 

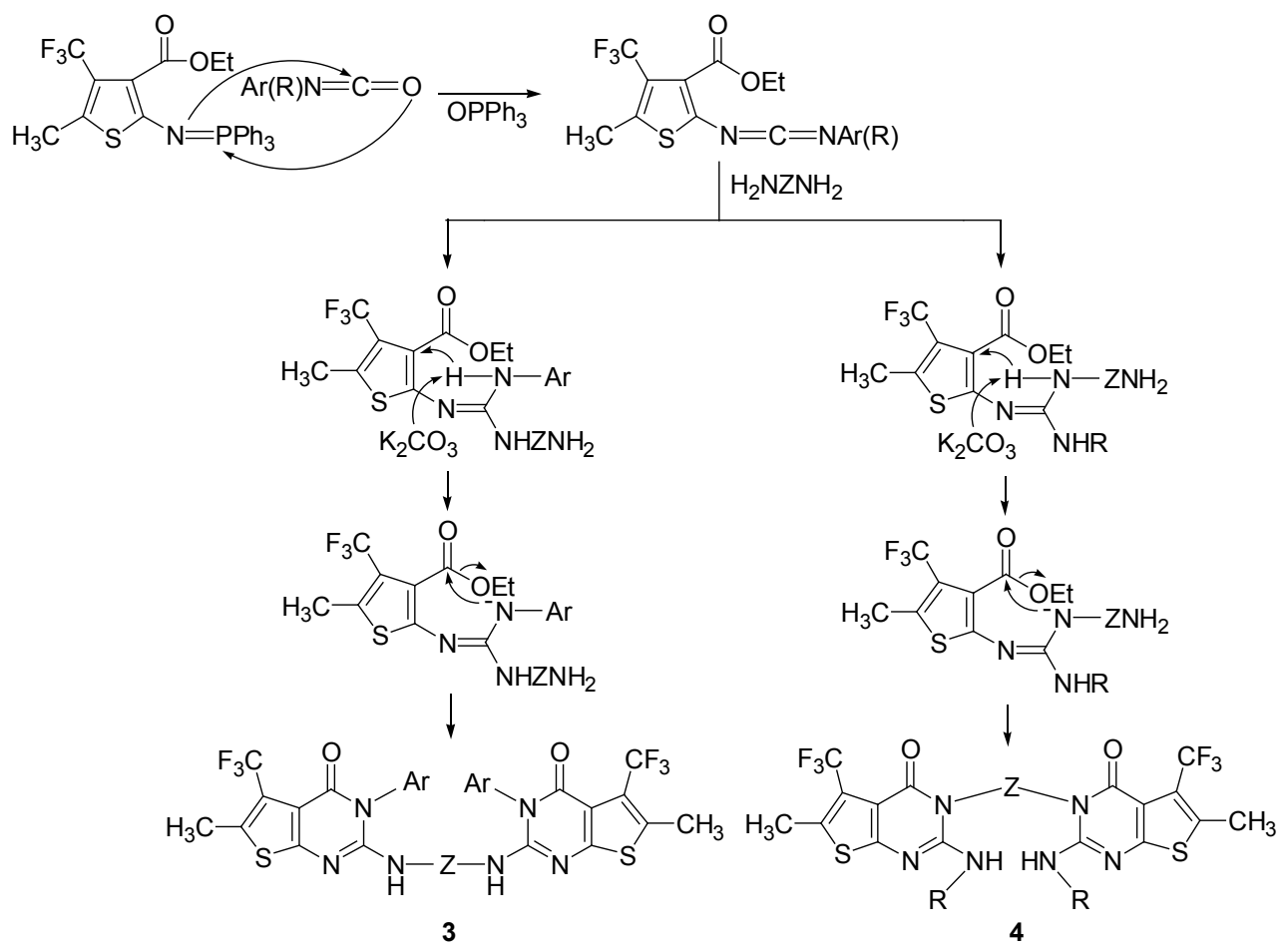

Scheme 3

$(\mathrm{NCH})$ 质子信号, 说明分子中存在 $\mathrm{CH}\left(\mathrm{CH}_{3}\right) \mathrm{NH}$. 化合物 4b 未见三重峰, 而是在 $\delta 3.32 \sim 3.33$ 表现为多重峰, 是 $\mathrm{NHCH}_{2}$ 中碳原子上的两个氢. 如果 $\mathrm{R}\left(\mathrm{CH}_{2} \mathrm{CH}_{2} \mathrm{CH}_{2} \mathrm{CH}_{3}\right)$ 在 3 位, 则会有 $\mathrm{CH}_{2} \mathrm{CH}_{2} \mathrm{CH}_{2} \mathrm{CH}_{3}$ 的第一位碳原子氢的三 重峰. 另外, 在 $\delta 4.32$ 处存在三重峰, 是 $\mathrm{NH}$ 基受邻近 $\mathrm{CH}_{2}$ 上氢原子裂分, 说明分子中存在 $\mathrm{NHCH}_{2} \mathrm{CH}_{2}$ $\mathrm{CH}_{2} \mathrm{CH}_{3}$ 基. 所有这些都证实桥联取代不在 3 位, 而是在 2 位. 当亲核试剂为对苯二胺时, 合成产物桥连苯环上 质子相互之间没有偶合, 化学位移在 7.5 附近, 呈现为 单峰.

在 IR 中, 标题物分子中嘧啶酮环的 $\mathrm{C}=\mathrm{O}$ 的强伸缩 振动峰出现在 $1660 \sim 1680 \mathrm{~cm}^{-1}$ 之间, 芳环上的 $\mathrm{C}=\mathrm{C}$ 双键的骨架伸缩振动在 $1381 \sim 1589 \mathrm{~cm}^{-1}$ 之间有 $2 \sim 3$ 个强弱不等的吸收峰. 环内 $\mathrm{C}=\mathrm{N}$ 双键的伸缩振动在 $1550 \sim 1572 \mathrm{~cm}^{-1}$ 之间. 特别是在 $3320 \mathrm{~cm}^{-1}$ 左右出现 $\mathrm{N}-\mathrm{H}$ 尖锐的特征吸收峰. 从其电离喷雾质谱数据可以 看出所有的化合物都有分子离子峰 $\left(\mathrm{M}^{+}+\mathrm{H}\right)$, 元素分析 测试结果也与计算值吻合, 这些特征都证实了合成化合 物的分子结构与预期相符.

\section{References}

[1] Chambhare, R. V.; Khadse, B. G.; Bobde, A. S.; Bahekar, R. H. Eur. J. Med. Chem. 2003, 38, 89.

[2] Ren Q. Y.; Liang, Y. J.; He, H. W.; Fu, L. W.; Gu, Y. C. Bioorg. Med. Chem. Lett. 2009, 19, 6713.
[3] Ren, Q. Y.; Cui, Z. P.; He, H. W.; Gu, Y. C. J. Fluorine Chem. 2007, 128, 1369 .

[4] Jennings, L. D.; Kincaid, S. L.; Wang, Y. D.; Krishnamurthy, G.; Beyer, C. F.; McGinnis, J. P.; Miranda, M.; Discafani, C. M.; Rabindran, S. K. Bioorg. Med. Chem. Lett. 2005, 15, 4731.

[5] Blanco, G.; Quintela, J. M.; Peinador, C. Tetrahedron 2008, 64, 1333.

[6] Bonne, D.; Coquerel, Y.; Constantieux, T.; Rodriguez, J. Tetrahedron: Asymmetry 2010, 21,1085.

[7] Palacios, F.; Alonso, C.; Aparicio, D. T.; Rubiales, G.; Santos, J. Tetrahedron 2007, 63, 523.

[8] Parmar, K.; Suthar, B.; Suthar, A.; Maheta, A. J. Heterocycl. Chem. 2009, 46, 975 .

[9] Hafez, H. N.; EI-Gazzar, A. B. A. Bioorg. Med. Chem. Lett. 2008, 18,5222 .

[10] Snegaroff, K.; Lasssagne, F.; Ababsa, G. B.; Nassar, E.; Ely, S. C. S.; Hesse, S.; Perspicace, E.; Derdour, A.; Mongin, F. Org. Biomol. Chem. 2009, 7, 4782.

[11] Pedeboscq, S.; Gravier, D.; Casadebaig, F.; Hou, G.; Gissot, A.; Giorgi, F. D.; Ichas, F.; Cambar, J.; Pometan, J. P. Eur. J. Med. Chem. 2010, 45, 2473.

[12] Yang, X. H.; Wu, M. H.; Sun, S. F.; Xie, J. L.; Ding, M. W.; Xia, Q. H. J. Heterocycl. Chem. 2008, 45, 1365.

[13] Fang, Z.-D.; Lü, J.-Q. Chin. J. Org. Chem. 2011, 31, 1888 (in Chinese). (方正东, 吕鉴泉, 有机化学, 2011, 31, 1888.)

[14] Liang, Y.; He, H. W.; Yang, Z. W. J. Heterocycl. Chem. 2011,48, 88.

[15] Song, A.; Lam, K. S. Tetrahedron 2004, 60, 8605.

[16] Yang, X. H.; Wu, M. H.; Ding, M. W. Chin. J. Org. Chem. 2010, 30, 1032 (in Chinese).

(杨绪红, 吴鸣虎, 丁明武, 有机化学, 2010, 30, 1032.) 